\title{
ARTCLE Gender dysphoria: treatment and outcomes ${ }^{\dagger}$
}

\author{
Kevan Wylie, Kate Eden \& Emily Watson
}

\begin{abstract}
Kevan Wylie is a Fellow of the Royal College of Physicians of London and the Royal College of Psychiatrists. He holds a Doctorate in couples sex therapy and has worked in sexual medicine since 1999. Dr Wylie is an Honorary Professor at Sheffield Hallam University and Associate Editor of the Journal of Sexual Medicine. He is a trustee of the patient charity the Sexual Advice Association. Kate

Eden is a Foundation Year 1 trainee at the Northern General Hospital,

Sheffield. She has a special interest in psychiatry, gender and sexual medicine, having studied under Professor Wylie as a medical student. Emily Watson is a

Foundation Year 2 doctor working in Sheffield. She has a special interest in psychiatry and sexual medicine, having studied during her elective under Professor Kevan Wylie. Correspondence Dr Kevan Wylie, Porterbrook Clinic, Nether Edge Hospital, Sheffield S11 9BF, UK. Email: k.r.wylie@sheffield.ac.uk
\end{abstract}

†See also pp. 2-11, this issue.

\begin{abstract}
SUMMARY
Once a formal diagnosis of gender dysphoria is established and comorbid disorders addressed, it is the role of the gender specialist to facilitate treatment according to agreed goals. This article reviews the treatment of gender dysphoria, including psychotherapy, and how eligibility and readiness for surgery are assessed. It considers issues facing transgender individuals detained in prison or psychiatric hospital, the factors that affect patients' satisfaction with gender reassignment surgery and how the specialist can ensure a satisfactory outcome.
\end{abstract}

\section{DECLARATION OF INTEREST}

None.

In the first of our articles on gender dysphoria in this issue (Eden 2012), we discussed recognition and assessment of the condition. Here we consider the role of the gender specialist in its treatment. The general goal of treatment in gender identity disorder is to allow the individual to find lasting comfort with their gendered self, thus maximising their overall psychological well-being and selffulfilment (World Professional Association for Transgender Health 2011). Some patients want only partial treatment (e.g. hormones alone) or to try to integrate both masculine and feminine aspects of themselves, with guidance from medical services. However, for the many patients whose goal is to 'change' their gender, 'triadic therapy' may be the desired treatment option.

\section{Triadic therapy}

Triadic therapy consists of:

- sustained experience of living in an identitycongruent gender role;

- administration of the hormones of the desired gender; and

- surgery to change the genitalia and other sexual characteristics.

It is the specialist's role to counsel the patient about the range of treatment options and their implications and limitations, to assess the patient's readiness to undertake each stage of therapy (although some patients will already have independently started living in their desired gender role or obtained hormones), to monitor, support and assist the patient throughout therapy and to engage the patient in psychotherapy.

\section{Living in an identity-congruent gender role}

Sustained experience living in the desired gender role or gender presentation (or evolving an already initiated change in gender role) is an important part of preparing for gender reassignment (World Professional Association for Transgender Health 2011). Specialists have an essential role in helping patients through this experience by providing them with information on how their legal name and gender can be changed and how to cope with appearing as the opposite gender in the workplace (Bockting 2008). The real-life experience is often misperceived by patients as being some kind of 'test' as to whether they will be 'allowed' surgery/ hormones and insistence on it by the specialist may result in conflict. Specialists can reassure patients that the exercise is designed as a chance to experience life and the issues involved in belonging to their preferred gender, to ensure that they are making an informed decision about their capacity to function in it (Bockting 2008).

\section{Hormone therapy}

When hormone therapy is started, it is the specialist's role to monitor the patient for any haematological, biochemical or physical consequences that this might cause (Wylie 2009). Specialists may also be required to refer patients to other services where available, for example dieticians, as a healthy weight is a prerequisite for most surgery. Speech therapy is important to change pitch and moderate resonance, and patients can be referred for this and for advice on non-verbal gestures and behaviours. Patients may also find the specialist useful for cosmetic advice, for example on hair removal, and for image advice. Many patients may not be aware that such services are available (Tugnet 2007).

\section{Psychotherapy}

Psychotherapy has been shown to be extremely beneficial for some patients with gender dysphoria 
and to have very positive outcomes. ${ }^{\ddagger}$ It is indicated as being essential in up to a third of patients (Seikowski 2007). It is important to note that the aim of psychotherapy is not to treat gender dysphoria in the sense of 'curing' patients of their 'misperceptions' about their gender. Rather, it is to help them to feel comfortable in their gender identity, allowing them realistic chances to succeed in relationships, education, and work (World Professional Association for Transgender Health 2011). This is achieved by enabling insight, working out coping strategies, giving psychological support and providing the patient with information about their treatment options. Many studies link psychotherapy with more favourable outcomes post-surgery (Bodlund 1996; Eldh 1997; Rehman 1999).

Part of the specialist's role is to give the patient 'permission' to explore their gender identity and sexuality in a non-judgemental, supportive atmosphere, as well as facilitating a 'coming-out' process. Keeping the 'secret' of one's transgender identity from others may contribute to emotional distress (Cole 1997), but disclosing it to all may lead to bullying.

Psychotherapy is usually on a one-to-one basis but some patients benefit from couple, family, group and relationship therapy. Including partners in therapy as early as possible results in a more favourable outcome (Bockting 2008). Involving parents may be helpful because the failure of parents to acknowledge the legitimacy of the patient's identity is viewed as an obstacle to the patient achieving a sense of self-acceptance (Nuttbrock 2002). Group therapy (and general support groups) offer the opportunity to foster peer support.

\section{Determining eligibility and readiness for gender reassignment}

\section{Eligibility}

It is the specialist's task to determine patients' eligibility and readiness for hormone therapy and surgery (World Professional Association for Transgender Health 2011; see also Eden 2012, this issue). A correct assessment of each individual is essential, because the aim of treatment is to improve their quality of life. Receiving treatments at the wrong time could be detrimental to the patient's social, mental and physical health.

The Standards of Care determine eligibility for gender reassignment in two parts: for the use of hormones and for surgery. The eligibility criteria for these are outlined in Box 1, but we recommend that readers consult the full document (World Professional Association for Transgender Health 2011: Appendix C).

\section{Readiness}

Readiness for treatment is based on both the specialist's and the patient's opinion. To be assessed as ready for hormone therapy, the patient must be judged by the specialist as likely to take hormones in a responsible manner. In addition, patients should demonstrate that they have further consolidated their preferred gender identity through experience living in an identity-congruent gender role or psychotherapy. As discussed in our previous article (Eden 2012, this issue), they should also have made progress in tackling any mental health or other identified problems (World Professional Association for Transgender Health 2011). The readiness criteria for genital surgery are similar: that progress has been made in consolidating the new gender identity and that comorbid problems have been addressed.

Specialists are reminded that the Standards of Care are guidelines only and are flexible (although if clinicians do modify them, they should explain this to the patient and obtain informed consent, to ensure both quality care and legal protection; World Professional Association for Transgender Health 2011). For example, patients can be given hormones before they begin living in their

BOX 1 Outline of eligibility criteria for gender reassignment treatment

Eligibility criteria for hormone treatment

1 Persistent, well-documented gender dysphoria;

2 Capacity to make a fully informed decision and to consent for treatment;

3 Age of majority in a given country (if younger, follow the SOC for children and adolescents);

4 If significant medical or mental concerns are present, they must be reasonably well-controlled.

Eligibility criteria for genital surgery

1 Persistent, well documented gender dysphoria:

2 Capacity to make a fully informed decision and to consent for treatment:

3 Age of majority in a given country:

4 If significant medical or mental health concerns are present, they must be well controlled;

5 Twelve continuous months of hormone therapy as appropriate to the patient's gender goals (unless the patient has a medical contraindication or is otherwise unable or unwilling to take hormones)

6 Twelve continuous months of living in a gender role that is congruent with their gender identity (in the case of metoidioplasty, phalloplasty and vaginoplasty)

(World Professional Association for Transgender Health 2011: Appendix C)
${ }^{\ddagger}$ Az Hakeem discusses psycho-

therapy for gender identity disorders on pp. 17-24, this issue. 
preferred gender identity, if hormone therapy will bring about changes in appearance that will facilitate integration into the chosen gender role in the workplace.

The eligibility and readiness requirements are also intended as minimum requirements. If the clinician is not sure that a patient is ready to progress to treatment and therefore wishes to delay the process, this delay should be incorporated into the treatment plan, with a full explanation (Meyer 2002).

\section{Additional criteria}

Change of name

Patients should change their name by a change of name document, a statutory declaration or deed poll. The patient should also be advised that they may be eligible and wish to apply for a Gender Recognition Certificate. However, this is not necessary for progression to surgery.

\section{Consent and capacity criteria}

If there are doubts about a person's capacity, the two-stage test of capacity (Mental Capacity Act 2005) must be recorded in the notes. First, is there an impairment of, or disturbance in, the functioning of the person's mind or brain? Second, if there is, is the impairment or disturbance sufficient that the person lacks the capacity to make the particular decision to proceed to gender confirmation surgery? A person is deemed to lack capacity to make a decision only if he or she is unable to: (a) understand the information relevant to the decision; (b) retain that information long enough to enable the making of a decision; (c) use or weigh that information as part of the process of making the decision; and (d) communicate the decision by any means.

\section{Special issues}

\section{Physical fitness to progress to surgical transition}

Three areas need attention. First, the patient should be advised to stop smoking. Second, they should be advised that weight limits might affect their eligibility for surgery: usually, a body mass index no higher than 31 and a waist circumference less than $100 \mathrm{~cm}$. The third issue concerns hair removal from the donor site. If a penoscrotal creation of a neovagina is likely to take place (i.e. the individual is circumcised), hair at the donor site should be removed by electrolysis or laser treatment over a period of 6 months before surgery, and it should be completed 6 weeks before surgery. If penile inversion is likely to proceed (i.e. if they are not circumcised), then hair removal is not necessary. There is no evidence that hair removal is essential and none of the procedures is permanent. However, there is some evidence that hair removal will reduce the likelihood of hair ball formation in the neovagina. With regard to transgender men, for pubic phalloplasty a phallus is created first before laser depilation. If radial forearm flap is used (from the non-dominant forearm), hair removal cream is advised 3 months before surgery.

\section{Detained transgender patients}

Transgender individuals may come into contact with mental health workers when detained, either in prison or as a psychiatric in-patient. They may also present voluntarily for in-patient psychiatric treatment. Whether or not a formal diagnosis of gender dysphoria is already established and therapy commenced, transgender individuals may have concerns about their treatment while away from their usual surroundings. These may include whether they will have access to therapies (including hormones) and to clothing appropriate to the pre-treatment experience of living in their identity-congruent gender role. Another common concern is whether they will be able to choose men's or women's accommodation. Concerns about accommodation relate not only to continuity of the perceived gender identity, but also to personal safety: there are frequent reports of attacks on transgender prisoners placed in prison accommodation unsuitable for their gender identity (Whittle 2007).

The current treatment of detained transgender people, especially in prison, appears inconsistent, with some countries providing better access to healthcare than others (Brown 2009). Although the sixth version of the Standards of Care was criticised for the brevity and content of its advice on the treatment of incarcerated people with gender identity disorder, this omission has been rectified in the latest version. In a chapter devoted to the subject clearly states that 'Health care for transsexual, transgender, and gender nonconforming people living in an institutional environment should mirror that which would be available to them if they were living in a noninstitutional setting within the same community'. (World Professional Association for Transgender Health 2011: p. 67)

\section{After treatment}

\section{Outcomes}

Although there is a paucity of long-term follow-up data that measure the true 'success' of hormonal and surgical treatment for gender dysphoria 
(Levine 2009), the consensus appears to be that the majority of patients who undergo gender reassignment surgery are satisfied with the outcome.

One study, however, found that $10 \%$ of patients show a worsening of their psychosocial condition after surgery (Michel 2001). Reasons suggested for poor post-surgical results are incorrect diagnosis, prior mental health problems and surgical complications (Decuypere 2006). Inadequate family support, weak social networks, unrealistic expectations of surgery and poor understanding of its limitations are also implicated as risk factors for post-surgical regret (Gorin 2008). Precisely which comorbid psychiatric conditions are risk factors for post-treatment regret is unclear as detailed data are lacking (Levine 2009).

It is tempting for both the patient and the clinician to view gender change (through surgery or hormones) as a 'solution' to all of the patient's problems, including comorbid psychopathologies. After all, the assumption that treatments that make the patient's anatomical gender more congruent with their gender identity will improve their quality of life is central to current management practice. In opposition, some clinicians argue that patients who have associated psychopathology before gender change will continue to be limited by these problems afterwards.

Levine \& Solomon (2009) point out that there is a distinct lack of comprehensive long-term followup studies on which to base either view. They present some experiences of patients with gender dysphoria post-hormonal/surgical therapy that illustrate the heterogeneity of treatment outcomes. Patients variously describe persistent significant regrets about transitioning, profound depression following reassignment surgery and, conversely, improved social functioning post-treatment.

\section{Follow-up care}

The role of the gender specialist in preventing and managing post-treatment regret lies in modifying risk factors for regret that have been identified. It is the specialist's responsibility to ensure that gender dysphoria is diagnosed accurately and any additional mental health problems are addressed before surgery being undertaken (Gorin 2008; see also Eden 2012, this issue). Specialists should make patients aware early on of the complications and risks involved in surgery, as well as highlighting that surgery has limitations and that the long-term outcomes of treatment are not fully known.

The gender specialist should be available for follow-up of previously seen gender patients, as this is one of the factors associated with good psychosocial outcomes (World Professional
Association for Transgender Health 2011; see also Eden 2012, this issue). Surgeons too, should make themselves available for follow-up care. It is particularly important that the patient has access to continuing psychotherapy, if required, because many find adjustment to their new gender difficult, the reactions of those around them may be upsetting and transitioning will not necessarily be the 'answer' to all of their problems. Once again it is worth emphasising that patients provided with the tools that create better mental health and increase resilience against adversity tend to fare better throughout life, regardless of the physical gender in which they live (Bockting 2007).

\section{Conclusions}

The specialist's role and addressing transgender issues from the perspective of the health professional and the patient

The specialist and patient should agree goals for treatment once a diagnosis of gender dysphoria is made. When the desired aim is to transition to the opposite sex, triadic therapy includes sustained experience of living in an identity-congruent gender role, hormonal treatment and genital surgery. It is the specialist's role to determine whether the patient is eligible and ready for treatment at each stage. Specialists should help the patient to consider the potential benefits that may follow from engaging in psychotherapy or other genderrelated counselling during the transitional stage.

Issues for detained transgender people include access to treatment (including the accoutrements that allow for the experience of living in the preferred gender identity) and suitable, safe accommodation. Access to these is inconsistent, and continuing input from mental health professionals on the needs of incarcerated transgender persons is required.

Some transgender patients express regret and dissatisfaction following genital surgery. The specialist can reduce this by establishing the correct diagnosis and by ensuring the patient's readiness for and understanding of the possible outcomes and limitations of surgery. Continuing care and post-surgical psychotherapy should be made available. There is a notable paucity of high-quality follow-up data regarding the outcomes of treatment for gender dysphoria.

Individuals with gender dysphoria have differing expectations and needs regarding support from professionals throughout transition, and an integrated multidisciplinary team is best placed to offer this. Transsexual, transgender and gender non-conforming people have many healthcare issues that go beyond hormone therapy and
MCO answers

$1 \mathrm{c} \quad 2$ e $3 d \quad 4$ b $5 d$ 
surgical intervention: the new ways of working encouraged in the seventh version of the Standards of Care (World Professional Association for Transgender Health 2011) will enable clinicians to support them in their lives.

\section{References}

Bockting W (2008) Psychotherapy and the real-life experience. From gender dichotomy to gender diversity. Sexologies 17: 211-24.

Bockting W, Knudson G, Goldberg J (2007) Counseling and mental health care for transgender adults and loved ones. International Journal of Transgenderism 9: 35-82.

Bodlund O, Kullgren G (1996) Transsexualism. General outcome and prognostic factors. A five-year follow-up study of nineteen transsexuals in the process of changing sex. Archives of Sexual Behavior 25: 303-16.

Brown G (2009) Recommended revisions to the World Professional Association for Transgender Health's Standards of Care section on medical care for incarcerated persons with gender identity disorder. International Journal of Transgenderism 11: 133-9.

Cole C, O'Boyle M, Emory L, et al (1997) Comorbidity of gender dysphoria and other major psychiatric diagnoses. Archives of Sexual Behavior 26: $13-26$

Decuypere G, Elaut E, Heylens G, et al (2006) Long-term follow-up. Psychosocial outcome of Belgian transsexuals after sex reassignment surgery. Sexologies 15: 126-33.

Eden K, Wylie K, Watson E (2012) Gender dysphoria: recognition and assessment. Advances in Psychiatric Treatment 18: 2-11.

Eldh J, Berg A, Gustafsson M (1997) Long-term follow up after sex reassignment surgery. Scandinavian Journal of Plastic and Reconstructive Surgery and Hand Surgery 31: 39-45.
Gorin A, Bonierbale M, Lancon C (2008) Gender dysphoria (GD). What role for the psychiatrist? Sexologies 17: S21.

Levine SB, Soloman A (2009) Meanings and political implications of 'psychotherapy' in a gender identity clinic. A report of 10 cases. Journal of Sex and Marital Therapy 35: 40-57.

Meyer W, Bockting W, Cohen-Kettenis P, et al (2002) The Harry Benjamin International Gender Dysphoria Association's Standards of Care for Gender Identity Disorders, Sixth Version. Journal of Psychology \& Human Sexuality 13: 1-30.

Michel A, Mormont C, Legros JJ (2001) A psycho-endocrinological overview of transsexualism. European Journal of Endocrinology 145 $365-76$

Nuttbrock L (2002) Transgender identity affirmation and mental health. International Journal of Transgenderism 6: 1-11.

Rehman J, Lazer S, Benet AE, et al (1999) The reported sex and surgery satisfactions of 28 postoperative male-to-female transsexual patients. Archives of Sexual Behavior 28: 71-89.

Seikowski K (2007) Psychotherapy and transsexualism. Andrologia 39: 248-52.

Tugnet N, Goddard JC, Vickery R, et al (2007) Current management of male-to-female gender identity disorder in the UK. Postgraduate Medical Journal 83: 638-42.

Whittle S, Turner L, Al-Alami M (2007) Engendered Penalties: Transgender and Transsexual People's Experiences of Inequality and Discrimination. Press for Change.

World Professional Association for Transgender Health (2011) Standards of Care for the Health of Transsexual, Transgender, and Gender Nonconforming People (7th version). WPATH (www.wpath.org).

Wylie K, Fung R, Boshier C, et al (2009) Recommendations of endocrine treatment for patients with gender dysphoria. Sexual and Relationship Therapy 24: 175-87.

\section{MCQs}

Select the single best option for each question stem

1 Patients with gender dysphoria should start hormone therapy:

a before beginning the experience of living in an identity-congruent gender role in most cases

b only if they wish to have surgery to change the genitalia and other sexual characteristics

c once both eligibility and readiness have been ascertained

$\mathrm{d}$ once eligibility has been ascertained

e once readiness has been ascertained.

\section{Considering patients post-gender} reassignment:

a more than $20 \%$ have ongoing gender dysphoric symptoms

b surgical complications occur for the majority of patients

c the psychiatrist should continue to be involved with family support for all patients

$\mathrm{d}$ patients should be assessed for post-surgical regret as a matter of routine

e long-term outcomes for this group of patients remain uncertain because of poor follow-up data.
3 According to the Standards of Care, to be eligible for genital surgery patients must:

a be over 18

b have a body mass index $<35$

c have applied for and obtained a Gender Recognition Certificate

d have had 12 months of continuous hormone therapy and experience of living in an identitycongruent gender role

e have completed at least 12 months of psychotherapy and experience of living in an identity-congruent gender role.

\section{As regards detained transgender} patients:

a the 6th edition of the Standards of Care comprehensively outlines how people with established gender identity disorders should be managed in prison

b patients presenting with new-onset gender identity disorders after the start of detention are not included in the 6th edition of the Standards of Care

c educating institutions about transgender prisoners is not the responsibility of the mental health professional d detained transgender patients should always be housed in the accommodation of their natal gender

e the incidence of attacks on transgendered prisoners is higher than any other minority group.

5 As regards triadic therapy:

a all patients with gender dysphoria aim to complete all stages of triadic therapy

b the specialist must prevent the patient from progressing to hormonal treatment if they decide the patient's experience of living in an identity-congruent gender role has not been sufficiently successful

c psychotherapy has been demonstrated to be essential in at least two-thirds of patients undergoing triadic therapy

$d$ the experience of living in an identity-congruent gender role is designed to allow patients to make informed decisions about their ability to function in their preferred gender

e it is not appropriate for the gender specialist to give information regarding legal matters such as changing name. 